\title{
Attending Health Care Provider
}

National Cancer Institute

\section{Source}

National Cancer Institute. Attending Health Care Provider. NCI Thesaurus. Code C81231.

The individual assigned to be the primary care manager overseeing a patient's medical care during an episode of hospitalization. 